\title{
Renal Liposarcoma in a Patient Undergoing Radiotherapy in Childhood: A Case Report
}

\author{
Pedro Nazareth Aguiar Jr ${ }^{\mathrm{a}, \mathrm{c}}$, Barbara de Souza Gutierres ${ }^{\mathrm{b}}$, Daiane Pereira Guimaraes ${ }^{\mathrm{a}}$
}

\begin{abstract}
Soft tissue sarcomas are rare neoplasms, which account for less than $1 \%$ of adult malignancies. In addition, they account for only $2 \%$ of renal cancers. The main mesenchymal lesions of the kidney are benign and difficult to differentiate with well-differentiated sarcomas. The aim of this report was to present the experience of the Hospital Sao Paulo (HU/UNIFESP) in a case of well-differentiated liposarcoma, which affected the kidney of a young adult who has been treated with radiotherapy in childhood, as well as to raise the relevant literature. A 26-year-old male patient with a history of prostate rhabdomyosarcoma that has been submitted to surgical resection and adjuvant radiotherapy 23 years ago in the childhood was presented. He was asymptomatic during 22 years, then he developed back pain, and underwent investigation and one tumor was detected in the right kidney. Radical nephrectomy was performed with complete resection of the lesion and he is currently without evidence of disease recurrence. Renal liposarcoma usually has good prognosis and the treatment is based almost exclusively on tumor resection. The differentiation between primary renal or retroperitoneal site is important for the prognostic assessment of the disease. There is a relationship between exposure to radiation in childhood and the development of second malignancy in adulthood, especially soft tissue sarcomas. Metastases are rare in well-differentiated forms of the disease. Recurrences can be observed in $30 \%$ of all cases and have been described after 13 years of initial diagnosis, which justifies the extended follow-up of these patients.
\end{abstract}

Keywords: Sarcoma; Liposarcoma; Kidney; Neoplasm; Case report

Manuscript accepted for publication August 27, 2014

${ }^{a}$ Centro de Quimioterapia da Universidade Federal de Sao Paulo, Escola Paulista de Medicina, Rua Pedro de Toledo, 377 Vila Clementino, Sao Paulo, SP, CEP 04039-030, Brazil

bUniversidade Paulista, Campus Tatuape, Rua Antonio de Macedo, 505, Parque Sao Jorge, Sao Paulo-SP, CEP 03087-040, Brazil

${ }^{\mathrm{c}}$ Corresponding Author: Pedro Nazareth Aguiar Junior, Centro de Quimioterapia da Universidade Federal de Sao Paulo, Escola Paulista de Medicina, Rua Pedro de Toledo, 377 Vila Clementino, Sao Paulo, SP, CEP 04039-030, Brazil. Email:pnajpg@hotmail.com

doi: http://dx.doi.org/10.14740/jmc1933w

\section{Introduction}

Soft tissue sarcomas are relatively rare neoplasms. They represent something around $0.8 \%$ of adults' neoplasms [1]. Liposarcoma is the third most commonly histology associated in the non-pediatric age (after GIST and undifferentiated sarcomas). It is described mainly on the extremities and retroperitoneum [1]. It is also known that only $1-2 \%$ of soft tissue sarcomas are primary of the kidney tissue and the most frequent renal sarcoma is leiomyosarcoma [2]. Differentiation between renal or retroperitoneal origin is crucial in defining therapeutic and prognostic of disease [3].

This is a case report study to report the experience of the Hospital Sao Paulo (HU/UNIFESP) in the treatment of a case of renal liposarcoma incident in a young adult patient.

\section{Case Report}

A 26-year-old male patient with a history of prostate rhabdomyosarcoma 23 years ago was presented. He does not have any familial history of genetic syndrome. Cystoprostatectomy was performed with neobladder construction. It was held adjuvant treatment with pelvic radiotherapy (we had lack of knowledge of previous dose). He remained asymptomatic until early 2013 when he developed pain in the right flank region. He showed neither weight loss nor hematuria. He did not present any kind of skin abnormalities.

We performed a computerized tomography which showed extensive lesion in the right kidney with fat density and destruction of the renal parenchyma (Fig. 1). Surgeons have opted for performing right nephrectomy that was performed in July 2013.

After surgical recovery, he was referred to outpatient follow-up and he has been being without evidence of disease recurrence at the last evaluation performed 10 months after surgery.

The pathological examination revealed a right nephrectomy product of $22.0 \times 15.0 \times 10.0 \mathrm{~cm}$. On sectioning, the kidney surface was yellow and softened with involvement of renal parenchyma and with the margins free of disease (Fig. 2). The final diagnosis was well-differentiated liposarcoma with areas of myxoid differentiation. It was noted renal parenchyma of $0.7 \mathrm{~cm}$ thick (Fig. 3, 4). 


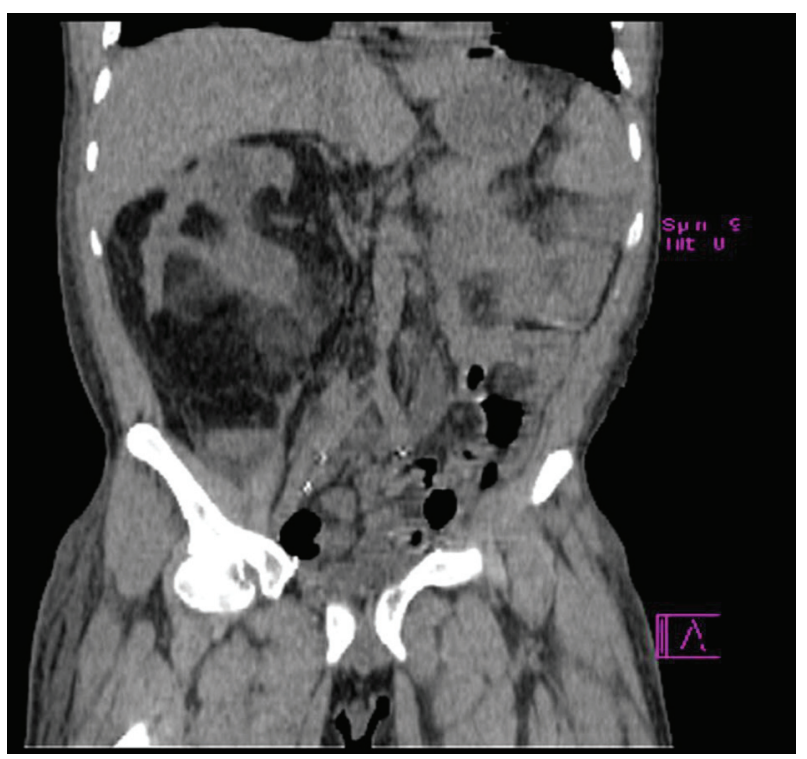

Figure 1. Computed tomography of the abdome showing a big mass in the region of the right kidney.

The patient remains without evidence of disease until the last evaluation 9 months after surgery.

\section{Discussion}

The exact incidence of mesenchymal tumors of the kidney remains undetermined; furthermore, almost all tumors are benign and asymptomatic [3]. One of the challenges for optimal therapeutic setting for these neoplasms is the rarity with which they are found. Sarcomas account for only $2 \%$ of malignancies of the kidney [4].

A study conducted at Memorial Sloan-Kettering Cancer Center has evaluated all 131 cases of sarcomas of the GU tract incidents between 1977 and 2003 in over 16 subjects; only 21 had renal location and of these only $26 \%$ were liposarcoma.

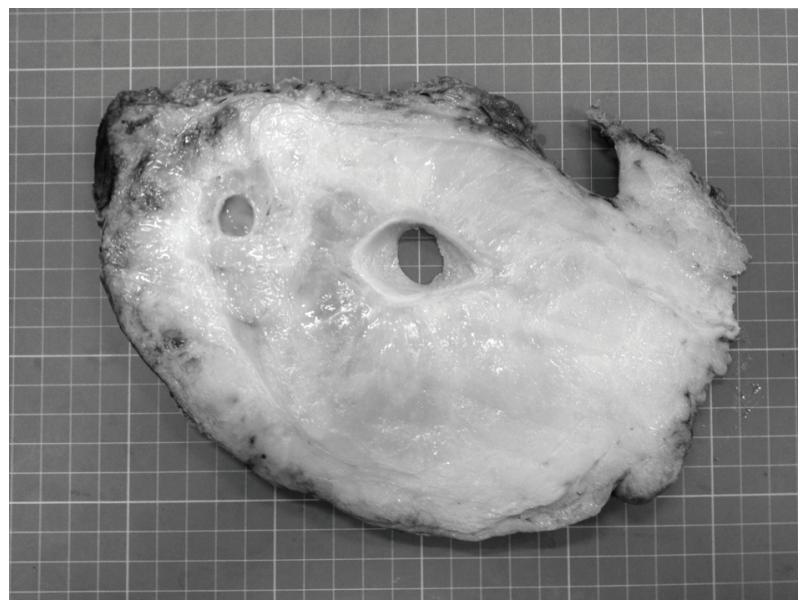

Figure 2. Kidney macroscopy.

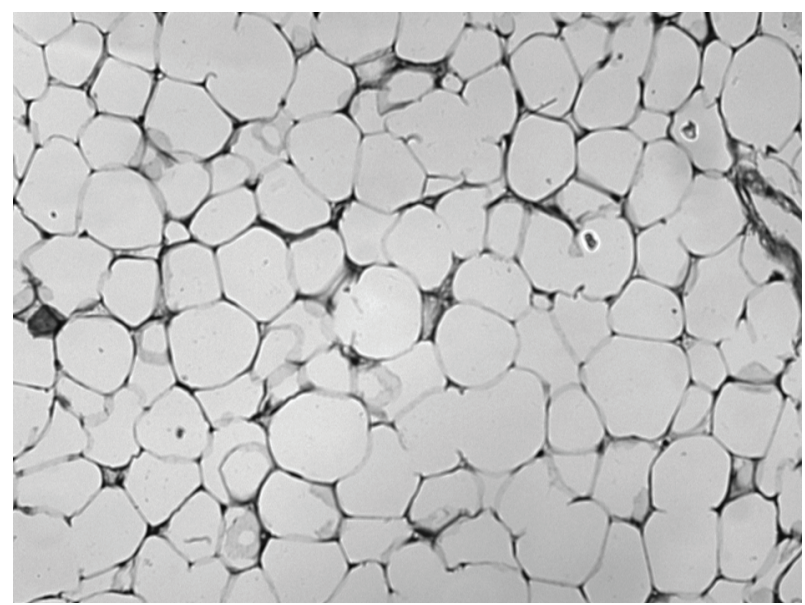

Figure 3. Hematoxylin and eosin in optical microscopy $(\times 200)$ showing lipoblasts with nuclear atypia and hyperchromasia.

The peak incidence of renal liposarcoma occurs between the fifth and sixth decades of life ( $76 \%$ of cases) and has a slight predominance in males. There is no preference for the right or left [5] kidney.

After identification of renal liposarcoma, it is important to differentiate between retroperitoneal or kidney disease therapy for the disease definition and prognosis. There are two criteria to confirm renal disease: parenchymal and unequivocal histological diagnosis of liposarcoma [6].

There are several morphological subtypes of liposarcomas and the identification is often difficult. It is known, however, that the majority $(40-45 \%)$ of the cases belong to the class of well differentiated. The lipoblasts are heterogeneous and they show focal nuclear atypia and hyperchromasia [7]. There are not genetic syndromes directly related to liposarcoma. It is known, nevertheless, that the myxoid and the round cell subtypes typically have the $\mathrm{t}(12 ; 16)$ translocation.

Exposure to chemotherapy (especially anthracyclines and alkylating agents) and the exposure to radiation can raise the

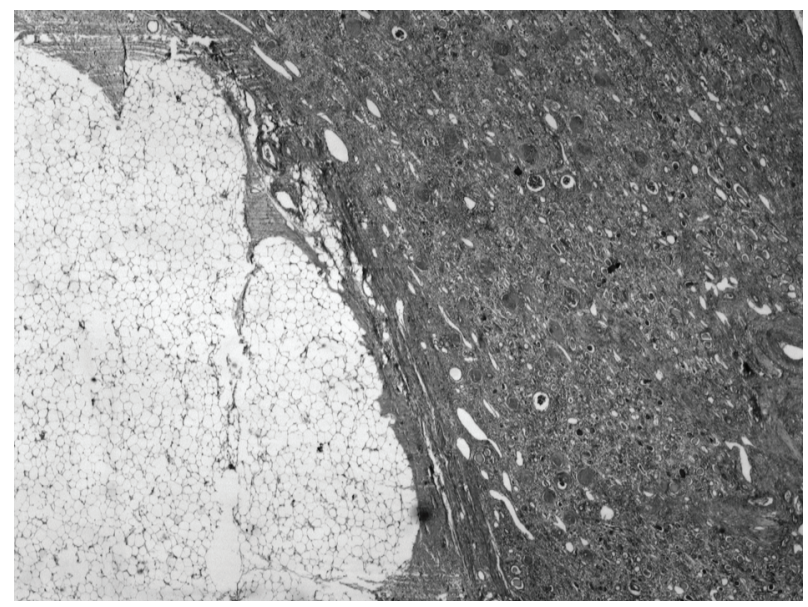

Figure 4. Hematoxylin and eosin optical microscopy $(\times 25)$ showing the interface between the tumor and the kidney parenchyma. 
risk of second malignancy, including soft tissue sarcomas. As an example, a British study that evaluates 16,541 survivors of childhood cancer showed incidence of 39 cases of soft tissue sarcomas when the value expected for the general population would be 1.1. The risk of developing a second neoplasia increases as higher as radiation dose and it also increases with the combination of chemoradiation. The adjusted risk in patients exposed to greater than 3,000 Gy was greater than 50 times higher than the population not exposed [8]. Our patient has had history of prior exposure to radiotherapy that may have contributed to a higher risk of developing second malignancy.

The main treatment described in the literature is nephrectomy and the prognosis depends on the degree of tumor differentiation, the size and the surgical margins free of neoplasia. When indicated, the chemotherapy is based on anthracyclines and the myxoid subtype has demonstrated statistically significant higher rate of response ( $48 \%$ vs. $18 \%$ ) compared to other liposarcoma subtypes [8]. However, low-grade tumors have shown higher survival global [9].

The prolonged follow-up is very important because there are reported cases with recurrence after 13 years of treatment [9]. Up to $30 \%$ of patients may develop local recurrence of the tumor and the risk is higher when lesions coexist in retroperitoneum or mediastinum. The liposarcoma has no metastatic potential when it is well differentiated; however, atypical forms have potential for distant metastases.

We conclude that renal liposarcoma is a rare tumor that needs to be differentiated from peritoneal liposarcoma which presents other behavior and prognosis. There is a relationship between radiotherapy in childhood and the incidence of second malignancy as soft tissue sarcoma. The treatment is based on surgical resection followed by prolonged clinical followup, because recurrences may occur late.

\section{Conflicts of Interest}

There are not any conflicts of interest.

\section{References}

1. Fletcher CDM, Bridge JA, Hogendoorn PCW, Mertens F. World Health Organization Classification of tumours of soft tissue and bone. 4th ed. Lyon: IARC Press; 2013.

2. Dotan ZA, Tal R, Golijanin D, Snyder ME, Antonescu C, Brennan MF, Russo P. Adult genitourinary sarcoma: the 25-year Memorial Sloan-Kettering experience. J Urol. 2006;176(5):2033-2038; discussion 2038-2039.

3. Algaba F, Moreno A, Trias I. Uropatologiatumoral. Tumoresmesenquimalesrenales. p. 80.

4. Russo P, Brady MS, Conlon K. Adulturological sarcomas. J Urol. 1992;174:1032.

5. Campbell's Urology. Sarcomas of the Kidney. 6th edition. 2(2): 1084 .

6. Laurino L, Furlanetto A, Orvieto E. Well diferenciated Liposarcoma (AtipicalLipomatousTumors). Seminars in Diagnostic Pathology. 2001;18:258.

7. Jenkinson HC, Winter DL, Marsden HB, Stovall MA, Stevens MC, Stiller CA, Hawkins MM. A study of soft tissue sarcomas after childhood cancer in Britain. Br J Cancer. 2007;97(5):695-699.

8. Jones RL, Fisher C, Al-Muderis O, Judson IR. Differential sensitivity of liposarcoma subtypes to chemotherapy. Eur J Cancer. 2005;41(18):2853-2860.

9. Mayes DC, Fechner RE, Gillenwater JY. Liposarcomarenal. Amer J SurgPathol. 1990;14:268-273. 